\title{
Aspremont, Garnier, La Bruyère, Voltaire, Corbière, Cendrars, dir. A. BOURMAYAN et G. VEYSSEYRE
}

\section{Roberta Sapino}

\section{OpenEdition}

\section{Journals}

\section{Edizione digitale}

URL: https://journals.openedition.org/studifrancesi/45620

DOI: 10.4000/studifrancesi.45620

ISSN: 2421-5856

\section{Editore}

Rosenberg \& Sellier

\section{Edizione cartacea}

Data di pubblicazione: 1 août 2021

Paginazione: 421-422

ISSN: 0039-2944

\section{Notizia bibliografica digitale}

Roberta Sapino, «Aspremont, Garnier, La Bruyère, Voltaire, Corbière, Cendrars, dir. A. Bourmayan et G.

VEYSSEYRE», Studi Francesi [Online], 194 (LXV | II) | 2021, online dal 01 septembre 2021, consultato il 15 octobre 2022. URL: http://journals.openedition.org/studifrancesi/45620 ; DOI: https://doi.org/10.4000/ studifrancesi.45620

Questo documento è stato generato automaticamente il 15 octobre 2022.

\section{(i) $\odot$

Creative Commons - Attribuzione - Non commerciale - Non opere derivate 4.0 Internazionale - CC BYNC-ND 4.0

https://creativecommons.org/licenses/by-nc-nd/4.0/ 


\title{
Aspremont, Garnier, La Bruyère, Voltaire, Corbière, Cendrars, dir. A. BOURMAYAN et G. VEYSSEYRE
}

\author{
Roberta Sapino
}

\section{NOTIZIA}

Aspremont, Garnier, La Bruyère, Voltaire, Corbière, Cendrars, dir. A. BOURMAYAN et G. VEYSSEYRE, Paris, Sorbonne Université Presses, 2019, 274 pp.

1 Inserito nella collana delle Sorbonne Université Presses intitolata «Styles, genres, auteurs», il volume curato da Anouch BOURMAYAN e Géraldine VEYSSEYRE è stato pensato così leggiamo nel sito della casa editrice - innanzitutto a vantaggio degli studenti di lettere che nel 2019 si stavano preparando alle prove dell'agrégation dell'anno seguente, ma intende raggiungere anche il pubblico più ampio degli studiosi che si interessano di stilistica.

2 La struttura è estremamente agile: sei brevissime sezioni («Aspremont», «Robert Garnier, Hippolyte», «Jean de La Bruyère, Les Caractères», «Voltaire, Zadig et L'Ingénu», «Tristan Corbière, Les Amours jaunes», «Blaise Cendrars, L'Homme foudroyé») senza alcuna introduzione o conclusione a inquadrarle ma seguite da una ricca bibliografia (pp. 249-265).

3 Apre il volume l'analisi condotta da François SUARD intorno alla rappresentazione della guerra nell'Aspremont: molteplici elementi, tra cui il ritmo narrativo sostenuto e l'importanza attribuita alla strategia nella battaglia, permettono di apprezzare l'originalità del testo rispetto ad altre chanson de geste tra cui la Chanson de Roland ( $L a$ guerre dans Aspremont, pp. 9-26). A seguire, Le vers d'intonation dans la chanson d'Aspremont, firmato da Muriel отт, propone una classificazione dei versi di intonazione presenti nell'Aspremont sulla base della distinzione tra récit e discours (pp. 27-50). 
4 In Lexique et poétique dans "Hippolyte": Garnier disciple de la Pléiade, Jean-Dominique BEAUDIN analizza i tratti lessicali salienti della tragedia umanista di Garnier (l'uso di parole rare, arcaismi, termini tecnici, per non menzionarne che alcuni) per dimostrare che l'autore si è appropriato delle teorie di du Bellay e di Ronsard con l'atteggiamento del "créateur intelligent" ben cosciente di star elaborando un'opera originale fondata sull'unione del tragico e dell'epico (pp. 55-66). Emmanuel BURoN smentisce poi il pregiudizio riguardo alla staticità delle tragedie umaniste: la loro spettacolarità, afferma lo studioso, deriva dalla messa in situazione di corpi parlanti. Con l'Hyppolyte, conclude, Garnier ha realizzato contemporaneamente "un poème dramatique et une pièce de théâtre" (L'écriture dramatique de Garnier dans "Hippolyte", pp. 67-87).

5 L'unico contributo dedicato a La Bruyère, firmato da Éric TOURRETTE e intitolato La subordination inverse dans "Les Caractères", si sofferma sulla subordinazione inversa: fatto linguistico che La Bruyère, appropriandosene nelle costruzioni a valore temporale, trasforma in tratto stilistico carico di espressività letteraria (pp. 91-105).

6 I due saggi dedicati a Zadig e a L'Ingénu si concentrano rispettivamente sull'humour, che Voltaire definisce in una lettera all'abbé d'olivet del 1762 e che assume valori di trasgressione diversi nei due testi (Violaine GÉRAUD, Les enjeux philosophiques de l'écriture humoristique dans "Zadig" et L"Ingénu", pp. 109-125), e sul legame - di cui è d'obbligo tenere conto per evitare di cadere nella semplice descrizione del fatto linguistico - che intercorre tra gli elementi sintattico-lessicali che compongono ogni opera e il quadro ideologico in cui essa si inserisce (Frank NEVEU, Voltaire et l'éthique de la langue. Petite cartographie de la phrase voltairienne dans "Zadig" et L"Ingénu", pp. 127-144).

7 Lo studio delle componenti di regolarità e irregolarità metrica in Les Amours jaunes conduce Benoît DE CORNULIER a concludere che Corbière, pur forzando a tratti la tradizione metrica, si inserisce nel suo solco, e ad affermare l'importanza, per chi si impegna nell'analisi ritmica delle sue poesie, di tener conto della coerenza interna di ciascun componimento ( $\mathrm{Du}$ jeu métrique dans "Les Amours jaunes" de Corbière, pp. 149-176). Corbière è stato, come illustra il contributo dal titolo evocativo di Tristan Corbière a donné sa langue au ÇA, il primo autore a usare la parola ça, peraltro facendone il titolo della prima sezione e della prima poesia di Les Amours jaunes, al di fuori degli spazi di riproduzione dell'oralità cui era confinata. Precursore - pur con le dovute differenze, che Benoît DUfAu illustra e analizza - delle riflessioni novecentesche sull'écriture blanche, Corbière fa prova, secondo lo studioso, di un intento preciso: «Contre le classique, il s'agit de faire valoir les droits de l'inclassable» (pp. 177-196).

Nell'ultima sezione, Karine GERMONI e Sandrine VAUDREY-LUIGI si concentrano su L'Homme foudroyé. La prima studiosa si sofferma sull'uso dei puntini di sospensione, tratto caratteristico dell'opera di Cendrars nel suo insieme, all'interno dei dialoghi tra personaggi, e ne identifica diverse funzioni: "phatique, prosodique, pantomimique, pragmatique, sémiotique» ( $\mathrm{Au}$ crible des points de suspension: les dialogues dans "L'Homme foudroyé" de Cendrars, pp. 201-229). La seconda inserisce l'analisi dell'espressione della vocalità che sottende L'Homme foudroyé all'interno di un discorso più ampio sul campo letterario nel quale il testo si inserisce (Phrase et oralité dans "L'Homme foudroyé": une phrase phonographe?, pp. 231-248). 